\title{
Can we REFINE stress-only SPECT MPI protocols using machine learning?
}

\author{
Mohamed Y. Elwazir, MD, ${ }^{\mathrm{a}, \mathrm{b}}$ and Panithaya Chareonthaitawee, $\mathrm{MD}^{\mathrm{a}}$ \\ a Department of Cardiovascular Medicine, Mayo Clinic, Rochester, MN \\ b Department of Cardiology, Faculty of Medicine, Suez Canal University, Ismailia, Egypt
}

Received Sep 21, 2021; accepted Sep 21, 2021

doi: $10.1007 / \mathrm{s} 12350-021-02822-4$

\section{See related article, pp. 2295-2307}

Single photon emission computed tomography (SPECT) myocardial perfusion imaging (MPI) is an indispensable tool for evaluation of patients with suspected or known coronary artery disease (CAD) given its noninvasive nature, widespread availability, and diagnostic and prognostic value. ${ }^{1}$ Protocols combining the assessment of both rest and stress SPECT MPI remain commonly utilized to detect and assess the burden of obstructive CAD. However, a strategy of stressfirst or stress-only MPI has been advocated by societies to reduce patient and personnel radiation dose and cost, and to improve laboratory efficiency and patient convenience. ${ }^{2}$ The safety of stress-only imaging has been shown in large clinical trials demonstrating comparably low event rates among normal studies using either stress-only or the combined stress/rest approach. Adoption of a stress-first over a rest-stress imaging protocol is further justified by temporal trends indicating that most patients with suspected or known CAD (without prior MI) referred for imaging will have a normal stress MPI. Despite their benefits, stress-first/ stress-only SPECT MPI protocols have not been widely adopted in the USA. The reasons for their significant underutilization are multifold but are also important for implementation of a successful stress-only imaging approach. The first consideration is patient selection. Despite the availability of published scoring systems for appropriate patient selection for stress-first/stress-only

Reprint requests: Panithaya Chareonthaitawee, MD, Department of Cardiovascular Medicine, Mayo Clinic, Rochester, MN; Chareonthaitawee. panithaya@mayo.edu

J Nucl Cardiol 2022;29:2308-10.

$1071-3581 / \$ 34.00$

Copyright (c) 2021 American Society of Nuclear Cardiology. protocols, there is lack of consensus in this regard. ${ }^{3,4}$ Most experts would agree that stress-only imaging should not be performed in those with known or highlikelihood of CAD, prior abnormal scans, or heart failure/reduced left ventricular systolic function. A BMI of greater than $35 \mathrm{~kg} / \mathrm{m}^{2}$ may be considered another criterion for avoiding stress-only imaging, though one study observed that only $22 \%$ of patients undergoing bariatric surgery (mean BMI $49 \mathrm{~kg} / \mathrm{m}^{2}$ ) required additional rest imaging. ${ }^{4}$ Another report indicated that lowdose and ultra, low-dose stress-only protocols should empirically be avoided in patients with BMI between 35 and $39.9 \mathrm{~kg} / \mathrm{m}^{2}$ and especially in BMI greater than 40 $\mathrm{kg} / \mathrm{m}^{2} .^{5}$ Recently, Rouhani et al published a clinical score model that included 9 variables (age in categories, typical angina, hyperlipidemia, current smoking, hypertension, diabetes, history of MI, history of percutaneous coronary intervention and history of coronary artery bypass grafting surgery). ${ }^{6}$ Depending on the sex of the patient, scores were assigned for the presence or absence of the variable, and a score of 0 to 1 was considered low risk for a non-normal MPI. While a step in the right direction, this model was derived in a singlecenter SPECT cohort and requires external validation. The model's area under the curve values were also only in the acceptable range (0.684 and 0.681$)$, raising concerns regarding the overall accuracy. It also requires experienced staff to carefully review the patient's chart and make determinations on chest pain characteristics and to tabulate all the variables and could be a barrier to implementation in busy practices. In addition, while the model can be used to predict those more likely to have a normal MPI, it does not identify those who may have artifacts or incomplete attenuation correction whereby resting images may still be required. The second barrier to implementation of stress-only/stress-first protocols is the need for rapid review of the stress images by an experienced reader to determine whether resting images should be performed. Delays in this step 
would mitigate the benefit of improving patient throughput with a stress-first/stress-only approach. This step is more challenging in busy practices or in laboratories without an on-site nuclear cardiologist. The third consideration for stress-only protocols is the need for high-quality images and experienced readers given the lack of resting images for comparison. Advanced SPECT technology including attenuation correction is even more essential and not consistently available in all laboratories and would reduce successful implementation of stress-first/stress-only protocols. Lastly, homogeneous perfusion throughout the myocardium, a criterion for a normal stress-first SPECT MPI, while clearly defined in published studies, is more elusive in real-life clinical practice. Given all of these factors to consider for successful implementation, it is not surprising that stress-first/stress-only SPECT MPI protocols are underutilized.

In this issue of the Journal of Nuclear Cardiology, Eisenberg and colleagues ${ }^{7}$ present a novel machine learning model trained to identify appropriate patients for stress-only MPI prior to physician review, and to predict the presence of obstructive CAD. The model included stress-only SPECT MPI automated total perfusion defect (TPD) values, wall motion and thickening, left ventricular volumes and ejection fraction, phase analysis parameters and stress ECG and pre-test clinical variables. This multicenter study used the REFINE SPECT registry ${ }^{8}$ which included 2,079 patients from 10 sites, utilizing 1,723 patients from 9 sites as the derivation cohort to train the model, which was then validated on the holdout set from the 10th site (356 patients), allowing external validation of their algorithm. The authors chose LogitBoost ${ }^{9}$ for their model; a member of the boosted, tree-based model family featuring automatic variable selection and pruning based on the additive value of each variable to the final model. This allowed the added benefit of identifying the subset of variables with actual predictive value for CAD, which are listed by the authors in order of importance. The model trained by Eisenberg et al. ${ }^{7}$ yielded a machine learning score (MLS), which had a consistently higher area under the curve (AUC) for CAD prediction than TPD or visual inspection by an expert, both on cross validation and in the hold-out population (0.82 vs. 0.74 vs. 0.68 , respectively). The authors experimented with several thresholds for the MLS and settled on a threshold of 0.29 , which had a sensitivity and specificity of $95 \%$ and $31 \%$, compared to $87 \%$ and $45 \%$, respectively, for $\mathrm{TPD} \geq 1 \%$ and $87 \%$ and $40 \%$, respectively, for visual interpretation. More importantly, since the ultimate purpose of the model is to effectively rule-out CAD to safely cancel rest imaging, the authors calculated the negative predictive value (NPV) of the MLS at the selected threshold to be $78 \%$, although they attribute this rather low value to the high prevalence of abnormal stress perfusion in the study cohort $(64.7 \%)$ compared to the entirety of the REFINE SPECT registry (25.5\%), and estimate that in a general patient population the NPV would be close to $95 \%$.

This novel model by Eisenberg et al. ${ }^{7}$ automates several challenging steps to implementation of a successful stress-first/stress-only SPECT MPI protocol, including patient selection and rapid image review. However, there are several interesting aspects of their cohort and approach that warrant further exploration. Prior publications and scoring systems for patient selection for stress-first/stress-only approaches have generally recommended that patients with known or high likelihood of CAD or with prior abnormal scans should not undergo stress-only imaging. In the current study, it appears that patients with a history of CAD, determined by a physician at each clinical site, were excluded but higher likelihood patients and those with prior abnormal scans were not. Regardless, the workflow in the current paper does require a manual review for patients with known CAD for exclusion and could slightly mitigate the benefits of their automated MLS.

Another confounding aspect of this study by Eisenberg et al. $^{7}$ is that the entire cohort comprised patients who have all undergone invasive coronary angiography (ICA). Because of the retrospective observational study design, these patients most likely had a high pre-test likelihood of CAD and higher risk to be referred for ICA. Because stress-only MPI generally should be avoided in patients with high likelihood of obstructive CAD, the type of patient represented by this cohort would normally not be eligible for stress-only MPI. This raises the question of whether the current results of the MLS are valid and/or generalizable to the lower likelihood cohort previously for stress-only MPI. In a prior publication, the same group reported superior mortality prediction with the MLS compared to readers while showing a similar proportion of rest scan cancellation between the MLS and non-MLS groups. ${ }^{10}$ The authors' rationale for including only patients with ICA in the current study's cohort was to ensure that coronary anatomy was accurately defined since SPECT MPI may be interpreted as normal in patients with high-risk CAD, including those with low pre-test likelihood of disease. It is notable that the MLS had higher area under the curve for CAD prediction than reader diagnosis or computergenerated TPD. Use of this information may ultimately aid the experienced reader in image interpretation but this remains to be tested in this cohort.

Although a multicenter study, all MPI acquisitions at all 10 participating sites by Eisenberg et al. ${ }^{7}$ were performed on high-efficiency, solid-state SPECT 
scanners in leading nuclear cardiology laboratories at major tertiary centers and practices. While solid-state SPECT systems are increasingly utilized around the world, they still comprise a relatively small proportion of SPECT cameras in clinical use worldwide. Whether this MLS model is generalizable to conventional SPECT technology is unknown. Furthermore, all 10 participating sites are tertiary academic centers with expertise in the practice of nuclear cardiology. Whether the MLS model applies to smaller, local community practices is also unknown. Lastly, a single commercial software program was used for image analysis and to quantify the TPD and other imaging measurements in the MLS, again limiting generalizability of the findings and approach.

Several prior reports on stress-only imaging have generally utilized attenuation correction algorithms. This study, on the other hand, did not use attenuation corrected images to generate the MLS. Interpretation of stress-only images as completely normal may be challenging without attenuation correction and the use of an MLS approach may be useful particularly in the latter.

Adoption of stress-first/stress-only imaging approaches has been slow due to the barriers discussed. Such approaches offer the advantage of reducing radiation exposure, improving laboratory throughput, reducing costs, and improving patient experience without compromising safety, diagnostic, or prognostic accuracy. Using imaging, stress, and pre-test clinical variables in a point-of-care automated machine learning approach with minimal physician intervention is a step in the right direction for laboratories, especially those with the same equipment and software as in the study and could help achieve the goal of implementing the stress-first/stress-only protocol.

\section{Disclosure}

The authors have no conflict of interest.

\section{References}

1. Wolk M, Bailey S, Doherty J, Douglas P, Hendel R, Kramer C, et al. ACCF/AHA/ASE/ASNC/HFSA/HRS/SCAI/SCCT/SCMR/
STS 2013 multimodality appropriate use criteria for the detection and risk assessment of stable ischemic heart disease: a report of the American College of Cardiology Foundation Appropriate Use Criteria Task Force, American Heart Association, American Society of Echocardiography, American Society of Nuclear Cardiology, Heart Failure Society of America, Heart Rhythm Society, Society for Cardiovascular Angiography and Interventions, Society of Cardiovascular Computed Tomography, Society for Cardiovascular Magnetic Resonance, and Society of Thoracic Surgeons. J Am Coll Cardiol 2014;63:4.

2. Dorbala S, Ananthasubramaniam K, Armstrong IS, Chareonthaitawee P, DePuey EG, Einstein AJ, et al. Single Photon Emission Computed Tomography (SPECT) myocardial perfusion imaging guidelines: Instrumentation, acquisition, processing, and interpretation. J Nucl Cardiol 2018;25:1784-846.

3. Gowdar S, Chaudhry W, Ahlberg AW, Henzlova MJ, Lane Duvall WL. Triage of patients for attenuation-corrected stress-first Tc99m SPECT MPI using a simplified clinical pre-test scoring model. J Nucl Cardiol 2018;25:4.

4. Gemignani AS, Muhlebach SG, Abbott BG, Roye GD, Harrington DT, Arrighi JA. Stress-only or stress/rest myocardial perfusion imaging in patients undergoing evaluation for bariatric surgery. $\mathrm{J}$ Nucl Cardiol 2011;18:886-92.

5. Thompson RC, O'Keefe JH, McGhie AI, Bybee KA, Thompson EC, Bateman TM. Reduction of SPECT MPI radiation dose using contemporary protocols and technology. JACC Cardiovasc Imaging 2018;11:282-3.

6. Rouhani S, Al Shahrani A, Hossain A, Yam Y, Wells RG, deKemp RA, et al. A clinical tool to identify candidates for stress-first myocardial perfusion imaging. JACC Cardiovasc Imaging 2020;13:10

7. Eisenberg E, Miller R, Hu L-H, Rios R, Betancur Acevedo J, Azadani P, et al. Diagnostic safety of a machine learning-based automatic patient selection algorithm for stress-only myocardial perfusion SPECT. J Nucl Cardiol 2021. https://doi.org/10.1007/ s12350-021-02698-4.

8. Slomka P, Betancur J, Liang J, Otaki Y, Hu L, Sharir T, et al. Rationale and design of the REgistry of Fast Myocardial Perfusion Imaging with NExt generation SPECT (REFINE SPECT). J Nucl Cardiol 2020;27:3.

9. Friedman J, Hastie T, Tibshirani R. Additive logistic regression: A statistical view of boosting (with discussion and a rejoinder by the authors). Ann Stat 2000;28:337-407.

10. Hu L, Betancur J, Sharir T, Einstein A, Bokhari S, Fish M, et al. Machine learning predicts per-vessel early coronary revascularization after fast myocardial perfusion SPECT: Results from multicentre REFINE SPECT registry. Eur Heart J Cardiovasc Imaging 2020;21:5.

Publisher's Note Springer Nature remains neutral with regard to jurisdictional claims in published maps and institutional affiliations. 